\title{
High-Performance Target Tracking Using Tracker Fusion in a Track-While-Scan Radar
}

\author{
Hazem Kamel $^{*}$ and K. H. Moustafa*
}

\begin{abstract}
In this paper, high-performance targets are tracked by fuzzy logic particle filter (FLPF) that uses fuzzy logic systems (FLS). It estimates the angular turn rate, which is included as a state component, and tunes dynamically the number of particles used to estimate the posterior distribution. A tracker fusion technique is proposed to reduce the computation load when the target is non-maneuvering by using the unscented Kalman filter (UKF) as it has less computational load compared to the particle filters. The UKF is known to be optimal and is employed for state estimation for linear and Gaussian systems. The proposed technique performed well when tracking a high-performance target. Moreover, the computation load was decreased due to the use of UKF when the target is moving in a straight-line motion.
\end{abstract}

Keywords: Particle filters, Fuzzy logic systems, Track-while-scan radar, high-performance targets.

\section{Introduction}

Target tracking is a hybrid estimation problem in which we predict the future trajectory of an object based on its previous states. A target such as a military aircraft may thrust, roll, and pitch; which results in a nonlinear model due to aircraft control and turbulence. The extended Kalman filter (EKF) and its higher orders are methods to solve such a problem. In the EKF, the real nonlinear models and non-Gaussian errors are approximated to linear and Gaussian in the neighborhood of the track [1,2]. An alternative to the EKF is to use the unscented Kalman filter (UKF) which was introduced in to offer superior performance to that of the EKF [3].

The UKF is optimal and is employed for state estimation for linear and Gaussian systems. The innovations from the UKF are used as test statistics, based on which hypothetical tests are carried out for maneuver detection. In target tracking, however, the actual measurement system is typically nonlinear and noise may exhibit non-Gaussian behavior. A strategy for estimating the target state in such a situation is to use the fuzzy logic particle filter (FLPF) [4].

\footnotetext{
* Egyptian Armed Forces
} 
The use of multiple trackers has many advantages over the system such as increased system reliability, robustness, and survivability. The main objective of tracker fusion is to reduce computation load. When the target is non-maneuvering, UKF tracker is used instead of the FLPF as the UKF has less computational load compared to the FLPF [5]. The UKF and FLPF are implemented at a Track-While-Scan (TWS) radar system to track the target. At each scan period, both trackers transmit binary data regarding a potential maneuver to the fusion center, where decision fusion is performed to detect the potential occurrence of the target maneuver. This problem setting brings new challenges. The problem of correlation between state estimates arises when several trackers carry out dynamic system monitoring and each tracker has its own data processing system. While the observation noise of each tracker can be safely assumed to be independent, the process noise is the same in the dynamic model. This assumption makes different state estimates correlated. However, in classical distributed detection when optimal decision rules are required, the input observations are generally assumed to be independent and identically distributed under each hypothesis. In dynamic systems, the situation is much more complicated. The distribution of observations under each hypothesis is not readily available, but evolves as the dynamics proceed. Except for linear Gaussian systems, the correlation between observations in a general dynamic system is difficult to characterize.

This paper is organized as follows. Formulating the problem is given in Section 2. A tracker fusion using a hysteresis loop is explained in Section 3. In Section 4, maneuvering target models are mentioned. The TWS radar using tracker fusion technique is explained in Section 5 followed by performance analysis and experimental results. Finally, a conclusion is given.

\section{Problem Formulation}

Let us assume the non-maneuvering target hypothesis to be $H_{0}$ and the maneuvering target hypothesis to be $H_{1}$ defined as follows:

$$
\begin{aligned}
& H_{0}: \mathbf{x}_{k+1}=F_{k}^{0} \mathbf{x}_{k}+\mathbf{v}_{k} \\
& H_{1}: \mathbf{x}_{k+1}=F_{k}^{1} \mathbf{x}_{k}+\mathbf{v}_{k}
\end{aligned}
$$

where $F_{k}^{l}$ is the state transition matrix and $\mathbf{v}_{k}^{l}$ is the process noise under the hypothesis $l$ at scan period $k$. Parallel distributed maneuver detection is shown in Figure 1.

In this block diagram, the target behavior is governed by the hypothesis $H_{0}$. At each scan period $k$, the tracker detects a jump from the normal model to a maneuver model based on its own observations as follows:

$$
d\left(u_{k}\right)_{<_{\delta_{k}=0}}^{\geq^{\delta_{k}=1}} \xi
$$

where $d($.$) is the fusion center decision statistic, u_{k}$ is the error between the target's predicted and actual position, $\xi$ is the corresponding threshold, and $\delta_{k}$ is the global decision at scan period $k$. The global decision is fed back to the trackers so they can either update the state distribution based on their own observations (when $\delta_{k}=0$ ) or announce the occurrence of target maneuver (when $\delta_{k}=1$ ). In case of non-maneuvering target, the UKF is used. Otherwise, the FLPF is used in a case of maneuvering target. 


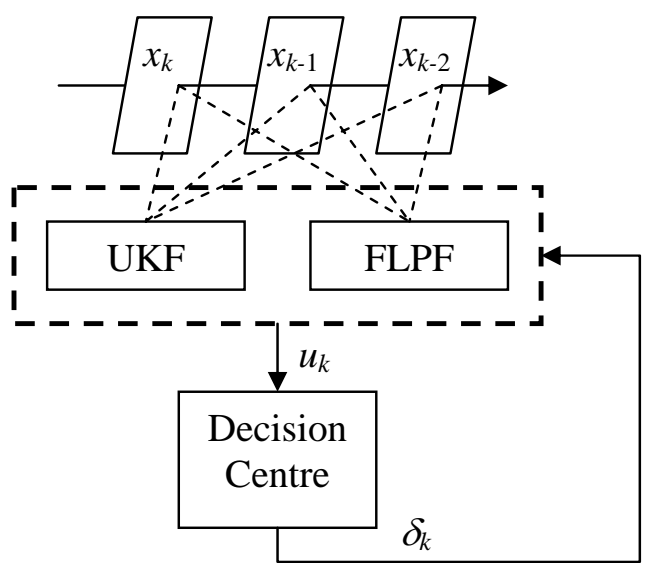

Figure 1. Maneuver detection block diagram.

Therefore, the tracker fusion can be explained as follows. Assume a target was detected for the first time at scan period $k-1$. The target's behavior would be governed by hypothesis $H_{0}$; i.e. non-maneuvering target. Consequently, the predicted target's state at scan $k$ would be calculated through the UKF tracker. Comparing the target's actual state to the predicted one at scan $k$, the error $u_{k}$ would be extracted. Comparing $u_{k}$ to the appropriate threshold corresponding to the antenna scan rate of the TWS radar, the target can be distinguished as maneuvering or not. If $u_{k}$ exceeds the threshold $\xi$, the target is considered maneuvering and the algorithm would switch to the FLPF tracker. Otherwise, the UKF would continue tracking the target.

\section{Tracker fusion using hystresis loop}

However, using one threshold $\xi$ may increase the computation load if the error varies in the neighborhood of the threshold. The MTT algorithm would switch from UKF to FLPF every time the error surpasses the threshold and vice versa. Therefore, a hysteresis loop is proposed as shown in Figure 2. If the target is moving in a straight line or slowly maneuvering, the UKF would be used. If the error $u_{k}$ exceeds a certain threshold $\xi_{2}$, the target would be considered maneuvering and the algorithm would switch to FLPF. The FLPF would track the target until the error goes below a threshold $\xi_{1}<\xi_{2}$. Obviously, the target would be either moving in a straight line or slowly maneuvering and the UKF is activated.

Therefore, if the error $u_{k}$ is between $\xi_{1}$ and $\xi_{2}$, the hypothesis used in the scan period $k$ would remain as it was in the scan period $k$ - 1 . If the error $u_{k}$ exceeds the threshold $\xi_{2}$, the system would be forced to follow hypothesis $\mathrm{H}_{1}$. Meanwhile if the error goes below the threshold $\xi_{1}$, the system would be forced to follow the hypothesis $\mathrm{H}_{0}$. 


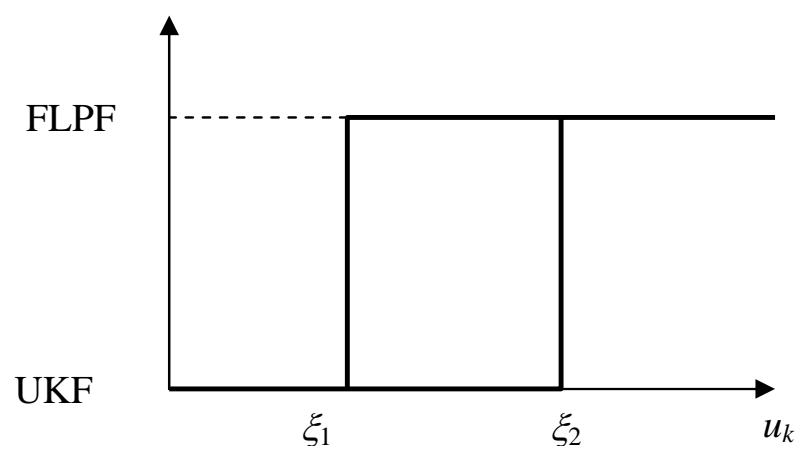

Figure 2. Tracker fusion using hysteresis loop.

To track multiple targets, the merged probabilistic data association (MPDA) approach [6] is used together with the UKF tracker. Meanwhile, assuming the observations are mutually independent on a scan-to-scan basis, the independent-sample based joint probabilistic data association (ISBJPDA) approach [7] is used together with the FLPF tracker.

\section{Maneuvering target model}

Maneuvers may be defined as a series of changes in direction and position for a specified purpose (as in changing course, switching tracks, or docking). Almost all maneuvering target tracking methods can be represented by some known mathematical models sufficiently accurately. The most commonly used such models are those known as state-space models, in form stated in Equation (4) with additive noise,

$$
\begin{aligned}
& X_{k+1}=F_{k}\left(x_{k}\right)+v_{k} \\
& z_{k}=H_{k} X_{k}+\omega_{k}
\end{aligned}
$$

One of the major challenges for target tracking arises from the target motion uncertainty. This uncertainty refers to the fact that an accurate dynamic model of the target being tracked is not available to the tracker.

Target motion models are normally classified into two classes: maneuvering and nonmaneuvering motion models. A non-maneuvering motion is the straight motion at a constant velocity, sometimes also referred to as the uniform motion. All other motions belong to the maneuvering mode.

Such motion is preferably specified in terms of the turn rate $\dot{\varphi}$. In the CT model with unknown turn rate, the turn rate is included as a state component, to be estimated. The FLPF is used to estimate the angular turn rate $\dot{\varphi}_{k}$ of the target. Consequently, the value of $\dot{\varphi}_{k}$ replaces $\dot{\varphi}$ in the transition matrix $F_{k}$. where: 


$$
\begin{aligned}
x_{k+1} & =F_{k}\left(x_{k}\right)+v_{k} \\
& =\left[\begin{array}{cccc}
1 & 0 & \frac{\sin \dot{\varphi} T}{\dot{\varphi}} & -\frac{1-\cos \dot{\varphi} T}{\dot{\varphi}} \\
0 & 1 & \frac{1-\cos \dot{\varphi} T}{\dot{\varphi}} & \frac{\sin \dot{\varphi} T}{\dot{\varphi}} \\
0 & 0 & \cos \dot{\varphi} T & -\sin \dot{\varphi} T \\
0 & 0 & \sin \dot{\varphi} T & \cos \dot{\varphi} T
\end{array}\right]\left(x_{k}\right)+v_{k}
\end{aligned}
$$

\section{TWS radar using tracker fusion}

Figure 3 shows the architecture of the proposed TWS radar using the tracker fusion technique explained previously. At scan period $k$, the targets' states are fed to both MPDA and ISBJPDA as well as the error calculation block. The actual targets' states $\left(\boldsymbol{x}_{k}\right)$ are assigned each to its corresponding track through the MPDA and ISBJPDA. Knowing the antenna scan rate, each target's state is compared to the predicted position calculated at the previous scan. In addition, the error calculation block generates the errors $u_{k}^{0}$ and $u_{k}^{1}$. Both errors are fed to the decision center to compare the error with the threshold $\xi_{k}$.

Based on the decision rule mentioned in Equation (3), a decision $\delta_{k}$ is taken whether the target of interest is maneuvering or not. The decision is fed to both trackers to decide which one should be used. If the target is a non-maneuvering target, the UKF tracker is used; otherwise, the FLPF is used.

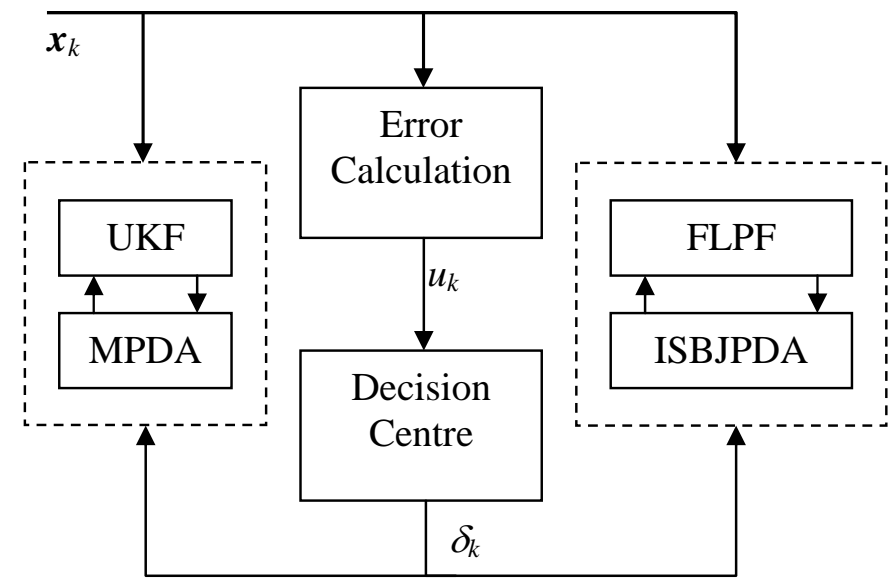

Figure 3. TWS radar with tracker fusion.

\section{Performance Analysis}

\section{Experiment Setup}

To simulate the backscattered signals from different aerial targets, the program package "Radar Target Backscattering Simulation" (RTBS) software [8] is used. The software package contains two programs: Target_editor.exe and BSS.exe. Different target models can be created or edited in Target_editor.exe. Meanwhile, the output of an amplitude or phase detector placed at the end of a linear receiver channel is calculated in the BSS.exe. The results 
of backscattered signals are saved in a data file that is processed by the main tracking program written in MATLAB 7.0. 500-Monte Carlo runs were used to get the experimental results. The probability of detection $\left(P_{d}\right)$ is set to be 0.9 and probability of false alarm $\left(P_{f a}\right)$ is $10^{-4}$.

Radar system

A 2D coastal radar system is chosen for the experiment. The radar pulse repetition frequency (PRF), $f_{r}$, is assumed to be $1100 \mathrm{~Hz}$. The antenna pattern has a "Cosine" amplitude distribution to provide low Side-Lobe Level (SLL). The transmitted pulse is a rectangular chirp frequency modulated to provide frequency agility against the foe's ECM. The pulse bandwidth is $200 \mathrm{MHz}$; meanwhile its width is $6 \mu \mathrm{s}$. The antenna aperture is $6 \mathrm{~m}$ and its scan period is chosen to be $5 \mathrm{~s}(12 \mathrm{rpm})$. Finally, the electromagnetic field is polarized vertically to omit backscattered pulses from sea waves.

Target model

a T-38 Talon trainer aircraft doing aerobatic trajectory is considered because it may reach a $13 \mathrm{~g}$ maneuver as it is not loaded with fuel or armaments. The T-38 Talon is a twin-engine, high-altitude, supersonic jet trainer used in a variety of roles because of its design, economy of operations, ease of maintenance, high performance and exceptional safety record. It is used primarily by Air Education and Training Command for undergraduate pilot and pilot instructor training. Student pilots fly the T-38 to learn supersonic techniques, aerobatics, formation, night and instrument flying and cross-country navigation. Consequently, the T-38 has more maneuverability than other fighters especially because it is not armed, which means it weighs less. Air Education and Training Command uses the T-38 Talon to prepare pilots for fighter aircraft such as the F-15.

\section{Experimental Results}

The UKF is set to be the TWS radar system default tracker. In this experiment, the T-38 Talon is performed a $13 \mathrm{~g}$ aerobatic trajectory. It was moving on a straight-line trajectory before starting to maneuver. The RMSE between the true and estimated position is shown in Figure 4 where the periods when the FLPF is active can be distinguished by shaded rectangles. When the T-38 starts to maneuver, the RMSE increases to $79 \mathrm{~m}$, approximately. When the RMSE surpasses the threshold $\xi_{2}$, the FLPF is activated and starts to track the maneuvering target. Consequently, the RMSE converges to its steady state and goes below the threshold $\xi_{1}$. Thus, the UKF is activated and starts to track the target when it is moving in a straight line till the next maneuver. Figure 5 shows the true and estimated trajectories. In this paper, the thresholds $\xi_{1}$ and $\xi_{2}$ are predefined to be $3 \mathrm{~m}$ and $40 \mathrm{~m}$, respectively. Also, the noise measurement is set to $50 \mathrm{~m}$ and the clutter density set to 0.02 . 


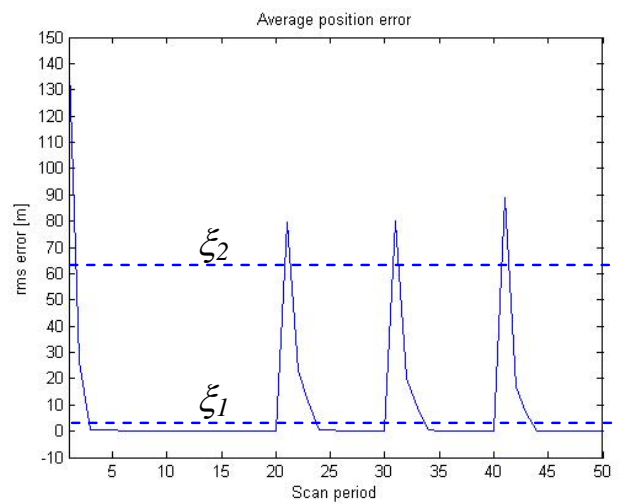

Figure 4. RMSE between predicted and estimated position of the T-38 Talon using the tracker fusion technique.

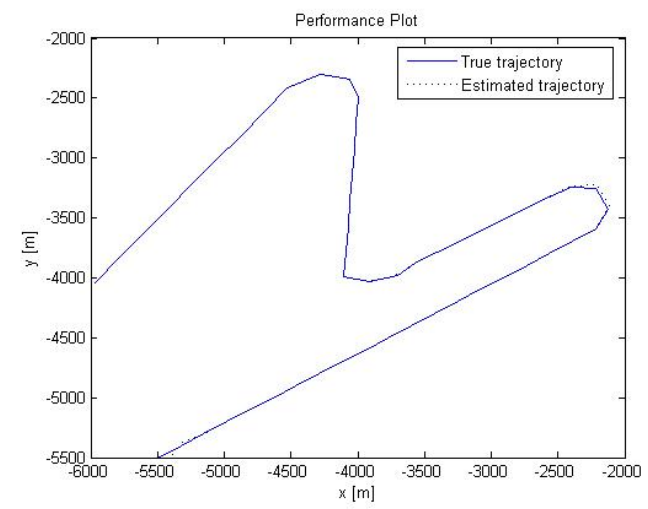

Figure 5. True and estimated trajectory of the T-38 using tracker fusion technique

\section{Conclusion}

In this paper, a new tracker fusion technique is introduced. Using tracker fusion, the maximum error in position does not exceed $90 \mathrm{~m}$ when a $13 \mathrm{~g}$ maneuver starts. This error converges to its steady state error within three scan periods. As well, the high-performance target's trajectory is tracked successfully using tracker fusion. The RMSE in case of using FLPF is less than that using tracker fusion. However, the main goal, which is tracking highperformance targets, is achieved by decreasing the computational load by using the UKF in tracking straight-line moving targets, slow-maneuvering targets, and high-performance maneuvering targets in the period that precedes the maneuver.

\section{References}

[1] S. Julier and J.K. Uhlmann, "A new extension of the Kalman filter to nonlinear systems," in Proc. of AeroSense: The $11^{\text {th }}$ International Symposium on Aerospace/Defence Sensing, Simulation and Controls, 1997.

[2] R. Wright, S.R. Maskell, and M. Briers, "Comparison of Kalman-based methods with particle filter for raid tracking," SEW Technology Domain, UK MoD Corporate Research Program, June 18, 2003. 
[3] E. A. Wan and R. van der Merwe, "The unscented Kalman filter”, in Kalman Filtering and Neural Networks, Chapter 7, Edited by Simon Haykin, Wiley Publishing, 2001.

[4] H. Kamel and W. Badawy, "A real-time multiple target tracking algorithm using merged probabilistic data association technique and smoothing particle filter," in Proc. $48^{\text {th }}$ Midwest Symposium on Circuits and Systems, Vol. 2, 2005, pp. 1537-1540.

[5] J.H. Kotecha and M. Djuri, “Gaussian particle filter," IEEE Trans. on Signal Processing, Vol. 51, No. 10, October 2003, pp. 2592-2601.

[6] H. Kamel and W. Badawy, "A suboptimal probabilistic data association technique for tracking multiple targets in dense clutter environment," in Proc. $5^{\text {th }}$ International Conference on Electrical Engineering, SP-1, May 2006, pp. 1-8.

[7] D. Schultz, W. Burgard, D. Fox, and A.B. Cremers, "Tracking multiple moving targets with a mobile robot using particle filters and statistical data association," IEEE International Conference on Robotics and Automation, 2001.

[8] S. Gorshkov, S. Leschenko, V. Orlenko, S. Sedyshev, and Y. Shirman, Radar Target Backscattering Simulation: Software and User's Manual, Artech House, 2002 